\title{
Factors Affecting CPAP Acceptance in Elderly Patients With Obstructive Sleep Apnea in Taiwan
}

\author{
Mei-Chen Yang MD, Chun-Yao Lin MD, Chou-Chin Lan MD PhD, Chun-Yao Huang MD, \\ Yi-Chih Huang MD, Chor-Shen Lim MD, Yu-Chih Liu MD PhD, and Yao-Kuang Wu MD
}

\begin{abstract}
BACKGROUND: The prevalence of obstructive sleep apnea (OSA) increases with age. Treatment often includes CPAP. CPAP adherence is correlated with disease severity and symptoms. We hypothesized that CPAP acceptance rates in elderly patients with OSA would be lower than in younger patients with OSA, and examined factors associated with CPAP acceptance. METHODS: We reviewed the charts of 315 subjects with OSA (apnea-hypopnea index $\geq 5$ events/h) who were treated at our hospital from 2008 to 2011 with CPAP therapy. All underwent CPAP titration testing before CPAP prescription. Subjects were grouped by age: young (25-40 y, $n=35)$, middle-age (41-65 y, $n=169$ ), and elderly ( $>65 \mathrm{y}, n=111$ ). Demographic variables, excessive daytime sleepiness, overnight polysomnography study variables, CPAP acceptance, and CPAP adherence were compared. Regression analysis was performed to identify factors associated with acceptance. RESULTS: The elderly subjects had less excessive daytime sleepiness and less pronounced alterations in overnight polysomnography variables than the subjects in the other groups, but had more cardiovascular comorbidities. The CPAP acceptance rate (overall 125/315, 39.7\%) was significantly lower in the elderly group, compared with the younger group $(31.5 \%$ vs $60 \%, P=.01)$. CPAP acceptance was associated with fewer comorbidities, higher excessive daytime sleepiness, and higher apnea-hypopnea index, but not age. CPAP adherence was not associated with age. CONCLUSIONS: CPAP acceptance is low in elderly patients in Taiwan. CPAP acceptance, instead of CPAP adherence, is the critical issue with elderly patients with OSA. Key words: elderly; obstructive sleep apnea; OSA; CPAP; adherence; acceptance. [Respir Care 2013;58(9):1504-1513. (C) 2013 Daedalus Enterprises]
\end{abstract}

\section{Introduction}

The prevalence of obstructive sleep apnea (OSA) increases with age. ${ }^{1-3}$ Physiological sleep alterations that increase with age include reduced sleep efficiency, delayed onset of sleep, less slow-wave sleep, a higher percentage of light sleep (stages 1 and 2), lower Epworth Sleepiness Scale scores, and less excessive daytime sleepiness. ${ }^{3-6}$

Drs Mei-Chen Yang, Chou-Chin Lan, Chun-Yao Huang, Yi-Chih Huang, Chor-Shen Lim, and Yao-Kuang $\mathrm{Wu}$ are affiliated with the Division of Pulmonary and Critical Care Medicine, Taipei Tzu Chi Hospital, Buddhist Tzu Chi Medical Foundation, New Taipei City, Taiwan. Drs Chun-Yao Lin and Yu-Chih Liu are affiliated with the Division of Pulmonary, Critical Care and Sleep Medicine, Chang Gung Memorial Hospital at Keelung, Keelung City, Taiwan. Drs Mei-Chen Yang, ChouChin Lan, and Yao-Kuang Wu are also affiliated with the School of Medicine, Tzu Chi University, Hualien, Taiwan.
These changes in sleep patterns may not be recognized as OSA because the symptoms of fatigue, hypersomnolence, and morning headache are often ascribed to other diseases or to aging itself. ${ }^{6}$ However, OSA, especially in the elderly, can reduce physical functioning in daily tasks, lessen memory recall, and increase the risk of fall and the associated consequences, including death. ${ }^{5}$ OSA is a significant risk factor for the occurrence of cardiovascular and cerebrovascular disorders. ${ }^{7}$ Simon et al recently suggested

\footnotetext{
The authors have disclosed no conflicts of interest.

Correspondence: Yao-Kuang Wu MD, Division of Pulmonary and Critical Care Medicine, Taipei Tzu Chi Hospital, the Buddhist Tzu Chi Medical Foundation, 289 Jianguo Road, Xindian District, New Taipei City 231, Taiwan. E-mail: drbfci@yahoo.com.tw.
}

DOI: $10.4187 /$ respcare. 02176 
that cardiovascular-related disease may indicate a higher risk for sleep apnea and that elderly patients with cardiovascular disease should be screened for sleep apnea. ${ }^{8}$

Interventions for OSA include weight loss, altered sleeping positioning, and CPAP. ${ }^{9}$ Elderly patients who use CPAP for OSA have increased alertness; improved neurobehavioral outcomes in cognitive processing, memory, and executive function; and decreased sleep disruption. ${ }^{10}$ However, many elderly patients have difficulty accepting and tolerating CPAP, and frequently cease CPAP use. ${ }^{11,12}$ CPAP cessation is also a problem in younger patients with OSA; indeed, there does not appear to be any marked difference in the rate of CPAP adherence in different age groups. ${ }^{11,13,14}$ Factors associated with poorer acceptance in the elderly include living alone, minor symptoms (especially hypersomnia), fewer skills or alteration of cognitive abilities, comorbidities, and neurological deficits. ${ }^{15}$

CPAP adherence in middle-age patients with OSA is associated with higher age, female sex, and lower Epworth Sleepiness Scale score. ${ }^{16}$ Adherence in elderly male patients improved with attendance at a CPAP education and support group, while nonadherence was associated with benign prostatic hyperplasia in elderly men. ${ }^{11}$ As the signs and symptoms of OSA in the elderly are less severe ${ }^{17}$ and the impact of the disease may be less pronounced ${ }^{18}$ than in younger individuals, we hypothesized that CPAP acceptance would be lower in elderly patients than in younger patients, and that different factors would be associated with CPAP acceptance and adherence in elderly patients. To test these hypotheses we retrospectively reviewed the medical records of 315 patients with newly diagnosed OSA who underwent polysomnography (PSG) to determine the rates of acceptance and adherence to CPAP and the independent factors associated with CPAP acceptance and adherence.

\section{Methods}

This retrospective review of medical records was approved by the institutional review board of Taipei Tzu Chi Hospital, the Buddhist Tzu Chi Medical Foundation, New Taipei City, Taiwan. Written informed consent was waived. Our chest clinic educates patients about OSA and CPAP therapy, and is involved in the monitoring and assisting of patients during treatment. We reviewed the records of 496 patients seen from January 2008 to December 2011, without previous diagnosis of OSA, who were referred from the chest clinic for PSG due to suspected OSA. Of these patients, 452 patients underwent overnight PSG (44 patients refused). Exclusion criteria for subsequent analysis included split-night PSG study $(n=47)$, lost to follow-up $(n=13)$, and normal PSG results $(n=69)$. Split-night PSG is ordered for patients who have severe signs and symptoms, suggesting severe OSA. As these

\section{QUICK LOOK}

\section{Current knowledge}

CPAP is an effective therapy for obstructive sleep apnea, but patient adherence to CPAP is poor. Cultural and age related factors in CPAP adherence have not been addressed.

\section{What this paper contributes to our knowledge}

In Taiwan, the rate of CPAP acceptance was very low in elderly patients with obstructive sleep apnea. Studies are needed to identify the key obstacles and solutions to CPAP acceptance among the elderly.

patients may therefore be more likely to accept CPAP therapy, they were excluded. In addition, split-night PSG can underestimate the apnea-hypopnea index (AHI). Patients with predominantly central apnea-hypopnea events were excluded. Eight of the 323 patients had mild OSA (AHI 5-15 events/h), but did not have excessive daytime sleepiness or cardiovascular complications, so CPAP was not required. The remaining 315 OSA patients (AHI $\geq 5$ events/h) who were symptomatic or had cardiovascular complications were given a CPAP titration test before CPAP was prescribed, and were included in the final analyses (Figure). These subjects were categorized into 3 age groups: young ( $n=35,25-40 \mathrm{y})$; middle-age $(n=169,41-65 \mathrm{y})$; and elderly $(n=111,>65 \mathrm{y})$.

\section{Assessment of Baseline Demographic and Clinical Characteristics}

Medical history and physical included weight, height, body mass index (BMI), neck circumference, waist and hip circumference, waist/hip ratio, smoking status, and comorbidities (hypertension, coronary artery disease, arrhythmia, cerebrovascular accident, COPD, asthma, and diabetes mellitus). Cardiovascular disease was defined as hypertension, coronary artery disease, or cerebrovascular accident. BMI values were categorized as either underweight $\left(<19.5 \mathrm{~kg} / \mathrm{m}^{2}\right)$, normal $\left(19.5-25.0 \mathrm{~kg} / \mathrm{m}^{2}\right)$, overweight $\left(25.0-30.0 \mathrm{~kg} / \mathrm{m}^{2}\right)$, or obese $\left(>30.0 \mathrm{~kg} / \mathrm{m}^{2}\right)$. Morning and evening blood pressure (BP) was monitored.

\section{Blood pressure measurement}

The BP was measured by the PSG technicians at the sleep center in the right arm using a standard mercury sphygmomanometer and optimal cuff $(17-\mathrm{cm}$ cuff for the obese patients, and 13-cm cuff for the non-obese patients). Evening BP was measured prior to sleep onset and 




Figure. Flow chart. OSA = obstructive sleep apnea. PSG = polysomnography.

morning BP was measured immediately after awakening, with the patients still in a supine position and still attached to all of the monitoring equipments. Prior to measurement of evening BP, patients stay in a supine position for at least 15 minutes. Two consecutive measurements were performed on each occasion and were averaged to obtain the evening and morning BP. Mean arterial BP was calculated in the usual manner from the systolic BP and diastolic BP.

\section{Assessment of Daytime Sleepiness}

Daytime sleepiness was subjectively evaluated using the Epworth Sleepiness Scale at the sleep center, before over- night PSG study. ${ }^{19,20}$ Excessive daytime sleepiness was defined as an Epworth Sleepiness Scale score of $\geq 11$.

\section{Overnight Polysomnography}

Trained sleep technicians performed a standard overnight PSG study for at least 6 hours, with standard monitoring, including electroencephalography (EEG), electroculography, chin and bilateral anterior tibialis surface electromyography, electrocardiography, airflow through the nose and mouth by thermister, thoracoabdominal movements by respiratory inductive plethysmography, position sensor on the respiratory inductive plethysmography, snore sensor, and oxygen saturation on pulse oximetry $\left(\mathrm{S}_{\mathrm{pO}_{2}}\right)$ simultaneously. All the technicians had received appropriate training from the Taiwan Society of Sleep Medicine and had at least 1 year of experience. PSG data were analyzed by manual scoring of every 30 -second epoch. Sleep stage was scored by trained sleep technicians according to the standard criteria of Rechtschaffen and Kales. ${ }^{21}$ Apnea events were categorized into obstructive apnea, central apnea, mixed apnea, or hypopnea events. An apnea event was defined as the $80 \sim 100 \%$ reduction of airflow for at least 10 seconds. An obstructive apnea was defined as the requisite reduction of $80 \sim 100 \%$ airflow for at least 10 seconds with continued respiratory effort recorded in the chest and abdomen movements channels. A central apnea was defined as a cessation of both airflow and respiratory effort for at least 10 seconds. A mixed apnea was defined by a period of both airflow and respiratory effort cessation, followed by a period of continued airflow cessation in spite of gradually increasing respiratory effort. A hypopnea event was defined as at least 50\% reduction of airflow for at least 10 seconds or at least $30 \%$ reduction of airflow for at least 10 seconds as compared to baseline and associated with at least $3 \%$ oxygen desaturation or with an EEG arousal. The AHI was calculated as the total number of apnea and hypopnea events per hour of sleep. The desaturation index was calculated as the number of desaturations $\geq 3 \%$ per hour of recording. Arousal index was calculated as the number of arousal events per hour. Sleep efficiency was defined as the fraction of total sleep time to total recording time. Sleep latency was defined as the time from lights off to the first identifiable sleep stage. Rapid eye movement (REM) latency was defined as the time from the first identifiable sleep stage to the first REM sleep.

\section{CPAP Titration Test}

Thirty-nine patients refused the CPAP titration, so CPAP titration was performed with 276 subjects. Mask fit was optimized for all subjects before the test. Pressure was adjusted to eliminate $95 \%$ of apneas, hypopneas, and snor- 
Factors Affecting CPAP Acceptance in Elderly Patients With Obstructive Sleep Apnea

Table 1. CPAP/BPAP Devices

\begin{tabular}{lll}
\hline \hline \multicolumn{1}{c}{ Manufacturer } & \multicolumn{1}{c}{ Model } & Type \\
\hline ResMed, San Diego, California & S8 Elite & CPAP \\
& S8 AutoSet Spirit & Auto-titrating CPAP \\
CPAP & Auto-titrating CPAP \\
& S9 Elite & BPAP \\
& S9 AutoSet & BPAP \\
Respironics, Murrysville, Pennsylvania & VPAP III ST & Auto-titrating CPAP \\
& VPAP IV ST & Auto-titrating CPAP \\
& REMstar Auto M with A-Flex 510M & BPAP \\
Fisher \& Paykel, Auckland, New Zealand & REMstar Auto M 501M & BPAP \\
& BiPAP Synchrony & Auto-titrating CPAP \\
Puritan Bennett, Pleasanton, California & BiPAP Auto M Series 701M & SleepStyle 250 Auto Series \\
Breas Medical, Mölnlycke, Sweden & SleepStyle 254 & CPAP \\
& GoodKnight 420E & Auto-titrating CPAP \\
& GoodKnight 420S & CPAP \\
& iSleep 20i & Auto-titrating CPAP
\end{tabular}

$\overline{\mathrm{BPAP}}=$ bi-level positive airway pressure

ing, and to achieve an oxygen saturation of $>90 \%$ and AHI $<5$ events/h in subjects during REM sleep in a supine position.

\section{CPAP Prescription and Follow-Up}

After determining the optimal CPAP pressure, CPAP was suggested and prescribed. Subjects had choice of 5 types of CPAP device, each of which has a built-in counter to measure adherence (Table 1). Subjects were assisted in device selection according to their individual needs and preferences. In this study, BPAP was prescribed if a subject: could not tolerate CPAP because of persistent nasal mask air leak, very high pressure, or discomfort exhaling against positive pressure; had concomitant nocturnal breathing disorders, including restrictive thoracic disorders, COPD, or nocturnal hypoventilation; or had central sleep apnea that was unresponsive to CPAP. After a subject accepted, purchased, and started long-term CPAP at home, they were followed-up after 1 month, and then every 3 months. Subjects brought their data card or CPAP equipment to each follow-up visit at our out-patient department, and adherence data were downloaded. Only the data from the last follow-up visit are presented in this study.

\section{CPAP Acceptance and Adherence}

CPAP acceptance was defined as CPAP use for at least the first 2 weeks. ${ }^{22}$ Patients who refused CPAP titration $(n=39)$, did not attend follow-up after CPAP titration $(n=24)$, refused CPAP after suggestion or prescription $(n=125)$, or chose positional therapy for their positional OSA $(n=2)$, were scored as non-acceptors of CPAP. CPAP adherence was defined as CPAP use for $\geq 4 \mathrm{~h} /$ night for $\geq 70 \%$ of the nights monitored, based on the built-in counter data from the last follow-up. 23,24

\section{Statistical Analysis}

The 3 continuous variables of neck circumference, waist/ hip ratio, and stage 2 sleep were normally distributed and are presented as mean $\pm \mathrm{SD}$. Normally distributed variables were compared by one-way analysis of variance. The remaining continuous variables were non-normally distributed and are presented as median and IQR values. Non-normally distributed variables were compared with the non-parametric Kruskal-Wallis test. Categorical variables are expressed as number and percentage, and were compared with the Fisher exact test. Bonferroni correction was used in the post-hoc tests for pair-wise group comparisons. Comparisons of pre-PSG and post-PSG systolic $\mathrm{BP}$, diastolic BP, mean BP within age groups were made using the nonparametric Wilcoxon signed-rank test. Univariable and multivariable logistic regression analyses were performed to evaluate the independent factors associated with CPAP acceptance and adherence. All significant factors identified in the univariable analyses, except for those that had collinearity with other variables, were entered into a multivariable logistic regression model. All statistical assessments were 2 -sided and were evaluated at the $P<.05$ level of statistical significance. Statistical analyses were 
Table 2. Demographics and Clinical Characteristics

\begin{tabular}{|c|c|c|c|c|}
\hline & \multicolumn{3}{|c|}{ Age Range, y } & \multirow[b]{2}{*}{$P$} \\
\hline & $\begin{array}{c}25-40 \\
n=35\end{array}$ & $\begin{array}{c}41-65 \\
n=169\end{array}$ & $\begin{array}{c}>65 \\
n=111\end{array}$ & \\
\hline Male, no. $(\%)$ & $33(94.3)$ & 135 (79.9) & $72(64.9)^{* \dagger}$ & $<.001$ \\
\hline Smoking, no. (\%) & & & & .01 \\
\hline Non-smoker & $17(48.6)$ & $79(46.7)$ & $48(43.2)$ & \\
\hline Past smoker & $3(8.6)$ & $54(32.0)^{*}$ & $39(35.1)^{*}$ & \\
\hline Smoker & $15(42.9)$ & $36(21.3)^{*}$ & $24(21.6)^{*}$ & \\
\hline Number of comorbidities, median (IQR) & $0(0-1.0)$ & $1.0(0-2.0)$ & $2.0(1.0-3.0) \ddagger \S$ & $<.001$ \\
\hline Cardiovascular disease, no. (\%) & $11(31.4)$ & $92(54.4)^{*}$ & $87(78.4)^{*} \dagger$ & $<.001$ \\
\hline Hypertension, no. (\%) & $11(31.4)$ & $84(49.7)$ & $83(74.8)^{* \dagger}$ & $<.001$ \\
\hline Coronary artery disease, no. $(\%)$ & $0(0)$ & $16(9.5)$ & $46(41.4)^{* \dagger}$ & $<.001$ \\
\hline Cerebrovascular accident, no. (\%) & $1(2.9)$ & $7(4.1)$ & $12(10.8)$ & .07 \\
\hline Arrhythmia, no. (\%) & $0(0)$ & $8(4.7)$ & $22(19.8)^{* \dagger}$ & $<.001$ \\
\hline COPD, no. $(\%)$ & $6(17.1)$ & $24(14.2)$ & $40(36.0) \dagger$ & $<.001$ \\
\hline Diabetes mellitus, no. (\%) & $2(5.7)$ & $25(14.8)$ & $30(27.0)^{* \dagger}$ & .005 \\
\hline Body weight, no. (\%) & & & & .02 \\
\hline Underweight: $\mathrm{BMI}<19.5 \mathrm{~kg} / \mathrm{m}^{2}$ & $0(0)$ & $1(0.6)$ & $4(3.6)$ & \\
\hline Normal weight: BMI $19.5-25.0 \mathrm{~kg} / \mathrm{m}^{2}$ & $6(17.1)$ & $46(27.2)$ & $34(30.6)$ & \\
\hline Overweight: BMI $25.0-30.0 \mathrm{~kg} / \mathrm{m}^{2}$ & $11(31.4)$ & $74(43.8)$ & $50(45.0)$ & \\
\hline Obese: $\mathrm{BMI} \geq 30.0 \mathrm{~kg} / \mathrm{m}$ & $18(51.4)$ & $48(28.4)^{*}$ & $23(20.7)^{*}$ & \\
\hline BMI, median (IQR) kg/m² & $30.1(27.1-33.8)$ & $27.3(24.7-30.4)^{*}$ & $26.4(24.2-2.0) \div$ & .001 \\
\hline Neck circumference, median (IQR) cm & $40.7 \pm 3.7$ & $39.2 \pm 3.4 *$ & $38.1 \pm 3.0 \dagger \neq$ & $<.001$ \\
\hline Waist circumference, median (IQR) $\mathrm{cm}$ & $102.0(92.0-111.0)$ & $96.0(91.0-103.0)$ & $98.0(90.0-105.0)$ & .09 \\
\hline Hip circumference, median (IQR) $\mathrm{cm}$ & $108.0(102.0-115.0)$ & $102.0(97.5-108.0) \ddagger$ & $100.0(96.0-108.0) \%$ & $<.001$ \\
\hline Waist/hip ratio, mean $\pm \mathrm{SD}$ & $0.938 \pm 0.054$ & $0.941 \pm 0.053$ & $0.959 \pm 0.060 \dagger$ & .02 \\
\hline 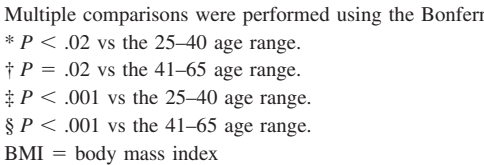 & ure with type-1 error adjustme & & & \\
\hline
\end{tabular}

performed with statistics software (SPSS 15.0, SPSS, Chicago, Illinois).

\section{Results}

\section{Baseline Demographic and Clinical Characteristics}

A total of 315 subjects with OSA were enrolled in the study, including $240(76.2 \%)$ males and 75 (23.8\%) females. The demographic and clinical characteristics of the subjects are summarized in Table 2. The 3 age groups significantly differed in sex, smoking history, and comorbidities. A significantly higher proportion of the elderly group had cardiovascular disease, including hypertension, coronary artery disease, and arrhythmia. Significantly more elderly subjects suffered from COPD and diabetes mellitus than did the other groups.

Physical characteristics were significantly different between the groups. Neck circumference was significantly lower in the elderly group, but BMI and the prevalence of obesity were significantly lower in the middle-age and elderly groups (see Table 2). Although hip circumference in the middle-age and elderly groups was significantly lower, the waist/hip ratio was significantly higher in the elderly group than in the middle-age group.

\section{Daytime Sleepiness and Overnight Polysomnography Results}

Daytime sleepiness and overnight PSG results differed with age (Table 3 ). The younger group had significantly higher Epworth Sleepiness Scale score, excessive daytime sleepiness, AHI, and desaturation index than did the middle-age and elderly groups. Significantly lower sleep efficiency and longer sleep latency were observed in the elderly group (see Table 3). Significantly higher stage 1 sleep, lower stage 2 sleep, and lower mean $\mathrm{S}_{\mathrm{pO}_{2}}$ were observed in the elderly group than in the middle-age group. Stage 3-4 sleep was significantly lower in the elderly group than in the 2 other groups. 


\section{Factors Affecting CPAP Acceptance in Elderly Patients With Obstructive Sleep Apnea}

Table 3. Daytime Sleepiness, Overnight Polysomnography, and Acceptance of CPAP

\begin{tabular}{|c|c|c|c|c|}
\hline & \multicolumn{3}{|c|}{ Age Range, y } & \multirow[b]{2}{*}{$P$} \\
\hline & $\begin{array}{c}25-40 \\
n=35\end{array}$ & $\begin{array}{c}41-65 \\
n=169\end{array}$ & $\begin{aligned} & >65 \\
n & =111\end{aligned}$ & \\
\hline $\begin{array}{l}\text { Excessive daytime sleepiness (Epworth } \\
\quad \text { Sleepiness Scale score } \geq 11 \text { ), no. (\%) }\end{array}$ & $26(74.3)$ & $79(46.7)^{*}$ & $48(43.2)^{*}$ & .004 \\
\hline Epworth Sleepiness Scale score & $13.0(10.0-15.0)$ & $10.0(7.0-14.0)^{*}$ & $9.0(5.0-14.0) \dagger$ & $<.001$ \\
\hline \multicolumn{5}{|l|}{ Overnight polysomnography } \\
\hline Apnea-hypopnea index, events/h & $52.6(20.6-73.5)$ & $35.5(23.1-56.0)^{*}$ & $34.3(21.9-50.5)^{*}$ & .02 \\
\hline Desaturation index, events/h & $50.0(12.4-71.8)$ & $23.9(11.8-46.8)^{*}$ & $22.7(7.7-39.4)^{*}$ & .01 \\
\hline Arousal index, events/h & $58.0(23.0-78.0)$ & $43.0(26.0-63.0)$ & $43.0(28.0-60.0)$ & .16 \\
\hline Sleep efficiency, $\%$ & $88.0(80.0-91.0)$ & $84.0(75.0-90.0)$ & $68.0(53.0-81.0) \dagger \dagger$ & $<.001$ \\
\hline Sleep latency, min & $13.0(10.0-22.0)$ & $14.0(8.0-22.0)$ & $22.0(11.0-38.0)^{*}+$ & $<.001$ \\
\hline REM latency, min & $106.5(81.0-151.0)$ & $102.0(75.5-178.0)$ & $111.0(82.0-180.0)$ & .87 \\
\hline S1 stage, $\%$ & $28.0(15.0-49.0)$ & $27.0(17.0-38.0)$ & $34.0(23.0-50.0) \ddagger$ & .003 \\
\hline $\mathrm{S} 2$ stage, mean $\pm \mathrm{SD} \%$ & $52.0 \pm 14.0$ & $56.2 \pm 14.1$ & $50.5 \pm 16.4 \S$ & .006 \\
\hline S3-4 stage, $\%$ & $2.0(0.0-9.0)$ & $0(0-3.0)$ & $0(0-0) \dagger \S$ & $<.001$ \\
\hline Non-REM stage $\mathrm{S} 1+\mathrm{S} 2+\mathrm{S} 3-4, \%$ & $91.0(83.0-94.0)$ & $88.0(83.0-94.0)$ & $91.0(85.0-96.0)$ & .20 \\
\hline REM stage, $\%$ & $9.0(6.0-17.0)$ & $12.0(6.0-17.0)$ & $9.0(4.0-15.0)$ & .20 \\
\hline Mean $\mathrm{S}_{\mathrm{aO}_{2}}, \%$ & $92.0(88.0-95.0)$ & $93.0(90.0-95.0)$ & $91.0(90.0-93.0) \ddagger$ & $<.001$ \\
\hline Nadir $\mathrm{S}_{\mathrm{aO}_{2}}, \%$ & $79.0(65.0-83.0)$ & $81.0(73.0-86.0)$ & $81.0(76.0-86.0)$ & .14 \\
\hline CPAP acceptance, no. (\%) & $21(60.0)$ & $69(40.8)$ & $35(31.5)^{*}$ & .01 \\
\hline $\begin{array}{l}\text { Values are median (IQR), unless otherwise indicated. } \\
\text { Multiple comparisons were performed using the Bonferron } \\
\text { REM = rapid eye movement. } \\
* P<.02 \text { vs } 25-40 \text { y range. } \\
\dagger P<.001 \text { vs } 25-40 \text { y range. } \\
\ddagger P<.001 \text { vs } 41-65 \text { y range. } \\
\S P<.02 \text { vs } 41-65 \text { y range. }\end{array}$ & with type-1 error adjustmer & & & \\
\hline
\end{tabular}

Table 4. Summary of Evening and Morning Blood Pressure Measurements by Patient Age Group

\begin{tabular}{|c|c|c|c|c|}
\hline & \multicolumn{3}{|c|}{ Age Range, y } & \multirow{2}{*}{$P$} \\
\hline & $\begin{array}{c}25-40 \\
n=35\end{array}$ & $\begin{array}{c}41-65 \\
n=169\end{array}$ & $\begin{aligned} & >65 \\
n & =111\end{aligned}$ & \\
\hline \multicolumn{5}{|c|}{ Systolic blood pressure, $\mathrm{mm} \mathrm{Hg}$} \\
\hline Evening & $131.0(122.0-143.0)$ & $124.0(116.0-134.0)$ & $129.0(119.0-136.0)$ & .02 \\
\hline Morning & $132.0(123.0-141.0)$ & $126.0(119.0-137.0)$ & $131.0(121.0-141.0)$ & .06 \\
\hline$P$ & .81 & $.005^{*}$ & $.008^{*}$ & \\
\hline \multicolumn{5}{|c|}{ Diastolic blood pressure, $\mathrm{mm} \mathrm{Hg}$} \\
\hline Evening & $83.0(75.0-88.0)$ & $78.0(71.0-85.0)$ & $74.0(68.0-80.0) \dagger+$ & $<.001$ \\
\hline Morning & $84.0(76.0-96.0)$ & $82.0(75.0-91.0)$ & $79.0(72.0-85.0) \S \|$ & .002 \\
\hline$P$ & $.04 *$ & $<.001 \Phi$ & $<.001 \Phi$ & \\
\hline \multicolumn{5}{|c|}{ Mean blood pressure, $\mathrm{mm} \mathrm{Hg}$} \\
\hline Evening & $99.7(92.0-105.0)$ & $93.7(86.0-100.3)$ & $91.3(85.7-98.3) \S$ & .007 \\
\hline Morning & $99.7(90.7-112.0)$ & $97.3(90.3-106.3)$ & $97.3(89.3-102.3)$ & .23 \\
\hline$P$ & .10 & $<.001 \Phi$ & $<.001 \Phi$ & \\
\hline $\begin{array}{l}\text { Values are med } \\
* P<.05 \text { betw } \\
\dagger P<.001 \text { vs } \mathrm{t} \\
¥ P<.001 \text { vs } \mathrm{t} \\
\$ P<.02 \text { vs th } \\
\| P<.02 \text { vs the } \\
\| P<<.001 \text { betv }\end{array}$ & $\begin{array}{l}\text { rformed using the Bonferroni } \\
\text { thin age range. }\end{array}$ & h type-1 error adjustment. & & \\
\hline
\end{tabular}


Factors Affecting CPAP Acceptance in Elderly Patients With Obstructive Sleep Apnea

Table 5. Summary of the Factors Associated With CPAP Acceptance

\begin{tabular}{|c|c|c|c|c|}
\hline & \multicolumn{2}{|c|}{ Univariate Analysis } & \multicolumn{2}{|c|}{ Multivariate Analysis } \\
\hline & Odds Ratio* $(95 \% \mathrm{CI})$ & $P$ & Odds Ratio* $(95 \%$ CI) & $P$ \\
\hline \multicolumn{5}{|l|}{ Age range, $\mathrm{y}$} \\
\hline $25-40$ & Reference & & Reference & \\
\hline $41-65$ & $0.46(0.22-0.97)$ & .04 & $0.53(0.21-1.33)$ & .10 \\
\hline$>65$ & $0.31(0.14-0.67)$ & .003 & $0.41(0.14-1.21)$ & .10 \\
\hline \multicolumn{5}{|l|}{ Sex } \\
\hline Female & Reference & & & \\
\hline Male & $2.33(1.31-4.16)$ & .004 & $1.31(0.62-2.77)$ & .10 \\
\hline \multicolumn{5}{|l|}{ Smoking status } \\
\hline Non-smoker & Reference & & & \\
\hline Past smoker & $1.03(0.61-1.75)$ & .91 & & \\
\hline Smoker & $1.11(0.63-1.96)$ & .73 & & \\
\hline \multicolumn{5}{|l|}{ Number of comorbidities } \\
\hline None & Reference & & Reference & \\
\hline $1-2$ & $0.44(0.26-0.73)$ & .002 & $0.43(0.23-0.81)$ & .009 \\
\hline $3-6$ & $0.32(0.15-0.69)$ & .004 & $0.38(0.14-1.06)$ & .06 \\
\hline \multicolumn{5}{|l|}{ Work status } \\
\hline Unemployed & Reference & & & \\
\hline Retired & $0.84(0.16-4.53)$ & .84 & & \\
\hline Employed & $0.70(0.14-3.55)$ & .67 & & \\
\hline Homemaker & $0.38(0.07-2.09)$ & .27 & & \\
\hline $\begin{array}{l}\text { Excessive daytime sleepiness (Epworth } \\
\quad \text { Sleepiness Scale score } \geq 11 \text { ) }\end{array}$ & $1.93(1.22-3.05)$ & .005 & $1.95(1.14-3.33)$ & .02 \\
\hline \multicolumn{5}{|l|}{ OSA severity } \\
\hline Mild: AHI 5-15 events/h & Reference & & Reference & \\
\hline Moderate: AHI 15-30 events/h & $5.60(1.56-20.16)$ & .008 & $8.13(2.13-31.02)$ & .002 \\
\hline Severe: AHI $>30$ events $/ \mathrm{h}$ & $17.17(5.16-57.07)$ & $<.001$ & $18.29(5.07-66.02)$ & $<.001$ \\
\hline Body mass index & $1.02(0.97-1.07)$ & .45 & & \\
\hline Neck circumference & $1.08(1.01-1.16)$ & .02 & $0.96(0.87-1.07)$ & .47 \\
\hline Waist/hip ratio & $1.57(1.04-2.37)$ & .03 & $1.43(0.81-2.53)$ & .22 \\
\hline Sleep efficiency & $1.01(0.99-1.02)$ & .28 & & \\
\hline Sleep latency & $1.00(0.99-1.01)$ & .49 & & \\
\hline \multicolumn{5}{|l|}{ Sleep stage } \\
\hline S1 & $1.02(1.00-1.03)$ & .01 & $1.00(0.98-1.02)$ & .72 \\
\hline $\mathrm{S} 2$ & $0.99(0.98-1.01)$ & .42 & & \\
\hline S3-4 & $0.93(0.88-0.98)$ & .01 & $0.94(0.88-1.01)$ & .08 \\
\hline REM & $0.96(0.93-0.99)$ & .008 & $0.99(0.95-1.04)$ & .76 \\
\hline \multicolumn{5}{|c|}{$\begin{array}{l}\text { * The odds ratios for continuous variables indicate that the probability of accepting CPAP was increased (odds ratio }>1 \text { ) or decreased (odds ratio }<1 \text { ) by every } 1 \text { unit increase in the corresponding } \\
\text { variable, except for the odds ratio for waist/hip ratio, which indicates that the probability of accepting CPAP was increased by every } 0.1 \text { unit increase in waist/hip ratio. } \\
\text { OSA = obstructive sleep apnea } \\
\text { AHI = apnea-hypopnea index } \\
\text { REM = rapid eye movement }\end{array}$} \\
\hline
\end{tabular}

\section{Blood Pressure Measurement}

There was a significant difference between morning and evening median diastolic BP in the younger group (Table 4). In the middle-age and elderly groups, median morning systolic BP, diastolic BP, and mean BP were all significantly higher than the corresponding evening values (see Table 4). Evening and morning diastolic BP were both significantly lower in the elderly group than in the other groups. Median evening mean BP, but not morning mean BP, was significantly lower in the elderly group than in the other groups.

\section{CPAP Acceptance}

A total of 125 subjects accepted CPAP, including 21 $(60.0 \%)$ in the younger group, $69(40.8 \%)$ in the middleage group, and $35(31.5 \%)$ in the elderly group. The proportion of subjects accepting CPAP was significantly lower in the elderly group than in the younger group 
Table 6. Summary of Modes and Adherence $(n=125)$

\begin{tabular}{|c|c|c|c|c|}
\hline & \multicolumn{3}{|c|}{ Age Range, y } & \multirow[b]{2}{*}{$P$} \\
\hline & $\begin{array}{c}25-40 \\
n=21\end{array}$ & $\begin{array}{c}41-65 \\
n=69\end{array}$ & $\begin{array}{c}>65 \\
n=35\end{array}$ & \\
\hline \multicolumn{5}{|l|}{ Mode, no. (\%) } \\
\hline Auto-titrating CPAP & $15(71.4)$ & $37(53.6)$ & $19(54.3)$ & .01 \\
\hline BPAP & $0(0)$ & $1(1.4)$ & $6(17.1)^{*}$ & \\
\hline CPAP & $6(28.6)$ & $31(44.9)$ & $10(28.6)$ & \\
\hline Follow-up duration, median (IQR) months & $5.5(3.0-13.0)$ & $10.5(3.5-15.5)$ & $9.0(3.0-16.0)$ & .50 \\
\hline Use, median (IQR) h/night $\dagger$ & $4.8(4.2-5.7)$ & $5.5(3.8-6.5)$ & $5.8(4.5-6.9)$ & .16 \\
\hline \multicolumn{5}{|l|}{ Adherence category, no. (\%) $\ddagger$} \\
\hline High & $11(52.4)$ & $45(65.2)$ & $24(68.6)$ & .46 \\
\hline Low & $10(47.6)$ & $24(34.8)$ & $11(31.4)$ & \\
\hline \multicolumn{5}{|l|}{ Continuity of use category, no. $(\%) \S$} \\
\hline Continuous & $15(71.4)$ & $46(66.7)$ & $26(74.3)$ & .77 \\
\hline Discontinuous & $6(28.6)$ & $23(33.3)$ & $9(25.7)$ & \\
\hline \multicolumn{5}{|c|}{$\begin{array}{l}* P=.02 \text { vs the } 41-65 \text { y range. } \\
\dagger \text { Determined from the data recorder of the CPAP or bi-level positive airway pressure (BPAP) device. } \\
\ddagger \text { High adherence }=\geq 4 \mathrm{~h} / \text { night for } \geq 70 \% \text { of the nights monitored. } \\
\S \text { Continuous use }=\text { every night. }\end{array}$} \\
\hline
\end{tabular}

(see Table 3). The main reasons for non-acceptance were cost, belief that the device is uncomfortable, and not believing there was a need for treatment of OSA.

\section{Factors Associated With CPAP Acceptance}

Univariable analyses revealed that age, sex, number of comorbidities, excessive daytime sleepiness, AHI severity, neck circumference, waist/hip ratio, stage 1 sleep, stage 3-4 sleep, and REM sleep were significantly associated with CPAP acceptance (Table 5). Most of these variables (age, sex, neck circumference, waist/hip ratio, stage 1 sleep, stage 3-4 sleep, and REM sleep) were not significantly associated with acceptance in the multivariable analysis (see Table 5). Multivariable analyses revealed that excessive daytime sleepiness, AHI severity, and the number of comorbidities were significantly associated with CPAP acceptance. After controlling for other factors in the multivariable analyses, subjects with 1-2 comorbidities were less likely to accept the CPAP than those with no comorbidities (odds ratio 0.43, $P=.009$ ). Conversely, subjects with excessive daytime sleepiness (Epworth Sleepiness Scale score $\geq 11$ ) were more likely to accept CPAP than subjects without excessive daytime sleepiness (odds ratio $1.95, P=.02$ ). Subjects with moderate and severe AHI were more likely to accept CPAP than those with mild AHI (odds ratios 8.13 and 18.29, respectively).

\section{CPAP Adherence}

The proportion of subjects using different modes differed with age. Specifically, BPAP use was significantly higher in the elderly than in the middle-age subjects (17.1\% vs $1.4 \%$ ) (Table 6). Of the 6 subjects who used BPAP in the elderly group, 3 could not tolerate CPAP because of the high pressure, one had severe mask leakage, one had moderate COPD with additional desaturation during sleep, and one had mixed obstructive and central sleep apnea due to congestive heart failure.

\section{Factors Associated With CPAP Adherence}

Univariable analyses revealed that smoking status was significantly associated with CPAP adherence; smokers had lower adherence than non-smokers (odds ratio 0.33, $P=.02$, Table 7).

\section{Discussion}

OSA can reduce physical functioning, lessen memory recall, and increase the risk of falling (and associated consequences, including death) in the elderly. ${ }^{5}$ In this study, we found that OSA subjects in the elderly group had fewer daytime symptoms, lower AHI, worse sleep efficiency, longer sleep latency, higher stage 1 sleep, lower stage 2 sleep, and lower mean $\mathrm{S}_{\mathrm{pO}_{2}}$ than did the middle-age and young subjects. These findings are consistent with those previously reported. ${ }^{4,5}$ The lower baseline $\mathrm{S}_{\mathrm{pO}_{2}}$ in the elderly subjects is interesting and may in part explain their less severe OSA symptoms. The lower baseline $\mathrm{S}_{\mathrm{pO}_{2}}$ may allow for easier attainment of the vertical part of the oxygen-hemoglobin dissociation curve and thus increased sensitivity to changes in oxygen and more rapid desaturation. In contrast, with the higher $\mathrm{S}_{\mathrm{pO}_{2}}$ in the younger 
Table 7. Summary of Factors Associated With CPAP Adherence

\begin{tabular}{|c|c|c|}
\hline & \multicolumn{2}{|c|}{ Univariate Analysis } \\
\hline & Odds Ratio* (95\% CI) & $P$ \\
\hline \multicolumn{3}{|l|}{ Age Range, y } \\
\hline $25-40$ & Reference & \\
\hline $41-65$ & $1.70(0.63-4.58)$ & .29 \\
\hline$>65$ & $1.98(0.65-6.05)$ & .23 \\
\hline \multicolumn{3}{|l|}{ Sex } \\
\hline Male & $0.42(0.13-1.36)$ & .15 \\
\hline Female & Reference & \\
\hline \multicolumn{3}{|l|}{ Smoking status } \\
\hline Non-smoker & Reference & \\
\hline Past smoker & $0.87(0.35-2.12)$ & .76 \\
\hline Smoker & $0.33(0.13-.82)$ & .02 \\
\hline \multicolumn{3}{|l|}{ Number of comorbidities } \\
\hline 0 & Reference & \\
\hline $1-2$ & $1.55(0.71-3.37)$ & .27 \\
\hline $3-6$ & $1.12(0.32-3.90)$ & .86 \\
\hline \multicolumn{3}{|l|}{ Work status } \\
\hline Unemployed & Reference & \\
\hline Retired & $7.00(0.54-91.11)$ & .14 \\
\hline Employed & $2.65(0.23-30.41)$ & .44 \\
\hline Homemaker & $8.67(0.58-130.11)$ & .12 \\
\hline $\begin{array}{l}\text { Excessive daytime sleepiness } \\
\quad \text { (Epworth Sleepiness } \\
\quad \text { Scale score } \geq 11)\end{array}$ & $1.04(0.50-2.18)$ & .92 \\
\hline \multicolumn{3}{|l|}{$\begin{array}{l}\text { Apnea-Hypopnea Index } \\
\text { severity }\end{array}$} \\
\hline Mild & Reference & \\
\hline Moderate & $7.5(0.53-105.28)$ & .14 \\
\hline Severe & $3.28(0.29-37.4)$ & .34 \\
\hline Body mass index, $\mathrm{kg} / \mathrm{m}^{2}$ & $0.96(0.89-1.04)$ & .32 \\
\hline Neck circumference, $\mathrm{cm}$ & $0.93(0.83-1.03)$ & .18 \\
\hline Waist/hip ratio & $1.37(0.66-2.85)$ & .59 \\
\hline Sleep efficiency, $\%$ & $1.01(0.98-1.03)$ & .37 \\
\hline Sleep latency, min & $0.99(0.98-1.01)$ & .14 \\
\hline \multicolumn{3}{|l|}{ Sleep stage, $\%$} \\
\hline S1 & $1.00(0.98-1.02)$ & .77 \\
\hline S2 & $1.01(0.99-1.04)$ & .30 \\
\hline S3-4 & $0.96(0.87-1.06)$ & .45 \\
\hline REM & $0.97(0.91-1.02)$ & .22 \\
\hline \multicolumn{3}{|c|}{$\begin{array}{l}\text { * The odds ratios for continuous variables indicate that the probability of CPAP adherence } \\
\text { was increased (odds ratio }>1 \text { ) or decreased (odds ratio }<1 \text { ) by every } 1 \text { unit increase in the } \\
\text { corresponding variable, except for the odds ratio for waist/hip ratio, which indicates that the } \\
\text { probability of accepting CPAP was increased by every } 0.1 \text { unit increase in waist/hip ratio. } \\
\text { REM = rapid eye movement }\end{array}$} \\
\hline
\end{tabular}

subjects, saturation is in the plateau part of the oxygenhemoglobin dissociation curve, rendering $\mathrm{S}_{\mathrm{pO}_{2}}$ less sensitive to changes in oxygen.

Due to these less severe symptoms in the elderly, we had hypothesized that the elderly subjects would have a lower CPAP acceptance rate than the younger subjects. Indeed, we did find this to be the case; CPAP acceptance was significantly lower in the elderly group than in the younger group. The overall rate of CPAP acceptance in our study was $39.7 \%$, which is lower than CPAP acceptance rates determined in studies in Europe and the United States, ${ }^{16,25,26}$ but much higher than in Poland (11\%). ${ }^{27}$ Interestingly, including our subjects who initially refused PSG or were not followed up $(n=57)$ reduced the acceptance rate to $33.6 \%$.

There are a number of possible reasons for the low CPAP acceptance in our cohort. First, healthcare in Taiwan is very accessible, which may cause some individuals to take their health for granted and not take the time to understand their illness. Such individuals want to be seen, diagnosed, and treated, but do not want to go to the trouble of using a CPAP machine. Second, CPAP is initiated at and all education conducted by a physician at the outpatient clinic. Educating patients about CPAP takes considerable time and effort, for which, unfortunately, physicians in Taiwan are not reimbursed, so the physicians cannot spend much time educating patients about the importance of treatment.

In a previous study, lower CPAP acceptance was associated with living alone, minor symptoms (especially hypersomnia), fewer skills, impaired cognitive abilities, comorbidities, and neurological deficits. ${ }^{15}$ In this study, greater CPAP acceptance was associated with fewer comorbidities, higher excessive daytime sleepiness, and higher AHI. Given these findings, it is unsurprising that the elderly group, who had more comorbidities and lower AHI and excessive daytime sleepiness, had a significantly lower acceptance rate than the other groups, who had higher AHI and excessive daytime sleepiness and fewer comorbidities.

One potentially confounding factor affecting CPAP acceptance is income. The national and commercial health insurance systems in Taiwan do not cover the cost of CPAP devices (U.S.\$1,500-3,000 in Taiwan). This is of interest, given the finding that supplying discounts on CPAP equipment may increase CPAP acceptance among patients of low socioeconomic status. ${ }^{28}$ Unfortunately, we were not able to obtain information on our subjects' income. Further study is needed to determine whether income affects CPAP acceptance.

Interestingly, our elderly subjects had significantly higher rates of BPAP use than the other groups. This may be because they had more comorbidities and were thus less able to tolerate CPAP. However, no subjects in the younger group used BPAP, so this finding must be interpreted with caution, and further study is needed.

The rate $(64 \%)$ of CPAP adherence in our cohort of Taiwanese subjects with OSA is similar to rates reported in previous studies. ${ }^{22,24,29,30}$ Further, the rate of CPAP adherence was similar for the 3 age groups. There was, however, a trend for higher adherence in the elderly subjects, particularly compared with the younger subjects. 


\section{Factors Affecting CPAP Acceptance in Elderly Patients With Obstructive Sleep Apnea}

This may reflect the fact that the elderly subjects had significantly more comorbidities and therefore may have been more concerned about their health than the healthier subjects in the younger group. However, neither comorbidities nor age were significantly associated with adherence. Indeed, the only factor associated with (poorer) adherence was smoking status. Clearly, further studies involving the assessment of other factors are needed to elucidate the mediators of patient adherence to CPAP.

\section{Conclusions}

The rate of CPAP acceptance was very low in our elderly subjects. Given the increasing number of elderly adults with OSA, healthcare professionals need to be aware of elderly sleep disturbances in order to better assess and treat these patients. Improving CPAP acceptance is paramount in managing OSA in elderly patients. Future studies are needed to identify the key obstacles and solutions to CPAP acceptance among the elderly. Patient-education programs that provide precise information about the risks of OSA and the benefits of treatment are needed.

\section{REFERENCES}

1. Setiati S, Laksmi PW. Insomnia in geriatrics. Acta Med Indones 2005;37(4):224-229.

2. Ancoli-Israel S, Kripke DF, Klauber MR, Mason WJ, Fell R, Kaplan O. Sleep-disordered breathing in community-dwelling elderly. Sleep 1991;14(6):486-495.

3. Neubauer DN. Sleep problems in the elderly. Am Fam Physician 1999;59(9):2551-2558.

4. Kobayashi M, Namba K, Tsuiki S, Matsuo A, Sugiura T, Inoue Y. Clinical characteristics in two subgroups of obstructive sleep apnea syndrome in the elderly: comparison between cases with elderly and middle-age onset. Chest 2010;137(6):1310-1315.

5. Neikrug AB, Ancoli-Israel S. Sleep disorders in the older adult-a mini-review. Gerontology 2010;56(2):181-189.

6. Wolkove N, Elkholy O, Baltzan M, Palayew M. Sleep and aging: 1. Sleep disorders commonly found in older people. CMAJ 2007;176(9): 1299-1304.

7. Banno K, Kryger MH. Sleep apnea: clinical investigations in humans. Sleep Med 2007;8(4):400-426.

8. Simon R, Chirakalwasan N, Teerapraipruk B, Hirunwiwatkul P, Jaimchariyatam N, Desudchit T, et al. Severity of obstructive sleep apnea in patients with and without cardiovascular-related diseases. Respir Care 2012;57(9):1476-1482.

9. Ballard RD. Management of patients with obstructive sleep apnea. J Fam Pract 2008;57(8 Suppl):S24-S30.

10. Weaver TE, Chasens ER. Continuous positive airway pressure treatment for sleep apnea in older adults. Sleep Med Rev 2007;11(2): 99-111.

11. Russo-Magno P, O’Brien A, Panciera T, Rounds S. Compliance with CPAP therapy in older men with obstructive sleep apnea. J Am Geriatr Soc 2001;49(9):1205-1211.
12. Wolkove N, Elkholy O, Baltzan M, Palayew M. Sleep and aging: 2. Management of sleep disorders in older people. CMAJ 2007;176(10): 1449-1454.

13. Parish JM, Lyng PJ, Wisbey J. Compliance with CPAP in elderly patients with OSA. Sleep Med 2000;1(3):209-214.

14. Sawyer AM, Gooneratne NS, Marcus CL, Ofer D, Richards KC, Weaver TE. A systematic review of CPAP adherence across age groups: clinical and empiric insights for developing CPAP adherence interventions. Sleep Med Rev 2011;15(6):343-356.

15. Lewis KE, Seale L, Bartle IE, Watkins AJ, Ebden P. Early predictors of CPAP use for the treatment of obstructive sleep apnea. Sleep 2004;27(1):134-138.

16. Sin DD, Mayers I, Man GC, Pawluk L. Long-term compliance rates to continuous positive airway pressure in obstructive sleep apnea: a population-based study. Chest 2002;121(2):430-435.

17. Russell T, Duntley S. Sleep disordered breathing in the elderly. Am J Med 2011;124(12):1123-1126.

18. Launois SH, Pepin JL, Levy P. Sleep apnea in the elderly: a specific entity? Sleep Med Rev 2007;11(2):87-97.

19. Johns MW. A new method for measuring daytime sleepiness: the Epworth sleepiness scale. Sleep 1991;14(6):540-545.

20. Chen NH, Johns MW, Li HY, Chu CC, Liang SC, Shu YH, et al. Validation of a Chinese version of the Epworth Sleepiness Scale. Qual Life Res 2002;11(8):817-821.

21. Rechtschaffen A, Kales AA. Manual of standardized terminology, techniques and scoring system for sleep stages of human subjects. Los Angeles: Brain Information Service/Brain Research Institute; 1968.

22. Anstead M, Phillips B, Buch K. Tolerance and intolerance to continuous positive airway pressure. Curr Opin Pulm Med 1998;4(6): 351-354.

23. Gay P, Weaver T, Loube D, Iber C; Positive Airway Pressure Task Force; Standards of Practice Committee; American Academy of Sleep Medicine. Evaluation of positive airway pressure treatment for sleep related breathing disorders in adults. Sleep 2006;29(3):381-401.

24. Kribbs NB, Pack AI, Kline LR, Smith PL, Schwartz AR, Schubert $\mathrm{NM}$, et al. Objective measurement of patterns of nasal CPAP use by patients with obstructive sleep apnea. Am Rev Respir Dis 1993; 147(4):887-895.

25. Collard P, Pieters T, Aubert G, Delguste P, Rodenstein DO. Compliance with nasal CPAP in obstructive sleep apnea patients. Sleep Med Rev 1997;1(1):33-44.

26. Waldhorn RE, Herrick TW, Nguyen MC, O’Donnell AE, Sodero J, Potolicchio SJ. Long-term compliance with nasal continuous positive airway pressure therapy of obstructive sleep apnea. Chest 1990; 97(1):33-38.

27. Frohnhofen H, Heuer HC, Kanzia A, Firat A. Influence of type of treatment for sleep apnea on activities of daily living in a sample of elderly patients with severe sleep apnea. J Physiol Pharmacol 2009; 60(Suppl 5):51-55.

28. Tarasiuk A, Reznor G, Greenberg-Dotan S, Reuveni H. Financial incentive increases CPAP acceptance in patients from low socioeconomic background. PLoS One 2012;7(3):e33178.

29. Meurice JC, Dore P, Paquereau J, Neau JP, Ingrand P, Chavagnat JJ, et al. Predictive factors of long-term compliance with nasal continuous positive airway pressure treatment in sleep apnea syndrome. Chest 1994;105(2):429-433.

30. Reeves-Hoche MK, Meck R, Zwillich CW. Nasal CPAP: an objective evaluation of patient compliance. Am J Respir Crit Care Med 1994;149(1):149-154. 